\title{
Seaweed floristic studies along tsunami affected Indian coasts: A litmus test scenario after 26th December 2004
}

\author{
VAIBHAV A MANTRI \\ Central Salt $\&$ Marine Chemicals Research Institute, Bhavnagar 364 002, Gujarat, India. \\ e-mail: vaibhav@csmcri.org
}

\begin{abstract}
On 26th December 2004, the world witnessed the devastating power of tsunami, affecting many countries, bordering the Indian Ocean region. This has caused significant changes in the shallow and intertidal regions of the Indian coast, especially the Andaman and Nicobar Islands, Tamil Nadu, Kerala and Pondicherry. The baseline data on biomass availability and distribution of benthic intertidal seaweed species were collected immediately after this catastrophic event by spot surveying 11 selected localities of the above-mentioned regions. In all, 45 species belonging to 31 genera were recorded during the present survey, the maximum number of seaweed species were recorded at Thirumullavarum, Kerala with the minimum at Car Nicobar, Andaman and Nicobar Islands. A very different trend was observed in the case of biomass availability at some locations which was due to the influence of habitat suitability over the tsunami damage. The details of this study have been provided in the present communication.
\end{abstract}

\section{Introduction}

The sheer power of nature unleashed the M9.0 earthquake of 26th December 2004 at 06:28:53 IST that had originated in the Indian Ocean just north of Simeulue Island, off the western coast of northwest Sumatra. This earthquake led to the devastating tsunami and created the most widespread catastrophe in modern history, particularly seen in Sri Lanka, India, Maldives, Indonesia and Thailand with damaging effects also in Malaysia, Bangladesh, Somalia and Seychelles. The huge tsunami waves caused mass movement of water with immense force, resulting in extensive damage to the nearshore property and major changes in the intertidal areas of the tsunami affected Indian coast (Jain et al 2005). Any assessment of the environmental impact of this cataclysmic tsunami will need both time and significant data.

India ranks first among all countries bordering the Indian Ocean ahead of Australia and South Africa (c.f. Sahoo et al 2001) in the number of recorded specific and intraspecific seaweed taxa.
However, information on the damage and changes to seaweed biota due to the recent tsumani along the Indian coast is not enough (Mantri 2005). Intertidal areas being more favourable for seaweed growth in the Indian waters, floristic studies for benthic, intertidal seaweeds were undertaken by the Central Salt and Marine Chemicals Research Institute, Bhavnagar during the period 15th January to 17th February 2005 along the tsunami affected coasts to assess what is left after 26th December 2004. The present communication is among the early reports about the impact of the recent tsunami on seaweed biota.

\section{Materials and methods}

Field observations were made during the lowest tide of the chart datum. Spot surveys were carried out along 11 localities (5 from Andaman and Nicobar Islands, 3 from Tamil Nadu, 2 from Kerala and 1 from Pondicherry) of tsunami affected Indian coast. All the seaweeds encountered during the

Keywords. Seaweeds; tsunami; species diversity; similarity index. 
Table 1. Checklist of seaweed species present along the tsunami affected Indian coast.

\begin{tabular}{|c|c|c|c|c|}
\hline \multirow{2}{*}{ Locality } & \multirow{2}{*}{$\begin{array}{c}\text { Date and time } \\
\text { of sampling }\end{array}$} & \multicolumn{2}{|c|}{ Seaweed encountered } & \multirow{2}{*}{$\begin{array}{l}\text { Availability } \\
\text { status }\end{array}$} \\
\hline & & Family & Species & \\
\hline \multirow{6}{*}{$\begin{array}{l}\text { South Point, Port Blair, } \\
\text { South Andaman Island } \\
11^{\circ} 39^{\prime} \mathrm{N} \& 92^{\circ} 45^{\prime} \mathrm{E}\end{array}$} & $\begin{array}{l}\text { 15th January } 2005 \\
06: 15 \mathrm{~h}\end{array}$ & Ulvaceae & $\begin{array}{l}\text { Ulva sp. } \\
\text { (Germling stage) }\end{array}$ & ++ \\
\hline & & & $\begin{array}{l}\text { Enteromorpha flexuosa } \\
\text { (Wulfen) J. Agardh }\end{array}$ & +++ \\
\hline & & Cladophoraceae & $\begin{array}{l}\text { Chaetomorpha_spiralis } \\
\text { Okamura } \\
\text { Cladophora sp. }\end{array}$ & $\begin{array}{l}+ \\
++\end{array}$ \\
\hline & & Dictyotaceae & $\begin{array}{l}\text { Lobophora variegata } \\
\text { (Lomouroux) } \\
\text { Womersley ex Oliveira } \\
\text { Padina tetrastromatica } \\
\text { Hauck }\end{array}$ & +++ \\
\hline & & Sargassaceae & $\begin{array}{l}\text { Sargassum cristaefolium } \\
\text { C. Agardh* } \\
\text { Turbinaria conoides } \\
\text { (J. Agardh) Kützing* } \\
\text { T. ornata } \\
\text { (Turner) J. Agardh* }\end{array}$ & $\begin{array}{l}+++ \\
++ \\
+++\end{array}$ \\
\hline & & Gracilariaceae & $\begin{array}{l}\text { Gracilaria canaliculata } \\
\text { Sonder }\end{array}$ & ++ \\
\hline $\begin{array}{l}\text { Malakka, Car Nicobar } \\
09^{\circ} 10^{\prime} \mathrm{N} \& 92^{\circ} 49^{\prime} \mathrm{E}\end{array}$ & $\begin{array}{l}\text { 21st January } 2005 \\
13: 30 \mathrm{~h}\end{array}$ & Ulvaceae & $\begin{array}{l}\text { Enteromorpha } \\
\text { compressa (Linnaeus) } \\
\text { Nees }\end{array}$ & +++ \\
\hline \multirow{3}{*}{$\begin{array}{l}\text { Diglipur, North Andaman } \\
\text { Island } \\
11^{\circ} 39^{\prime} \mathrm{N} \& 92^{\circ} 45^{\prime} \mathrm{E}\end{array}$} & $\begin{array}{l}\text { 29th January } 2005 \\
17: 00 \mathrm{~h}\end{array}$ & Dictyotaceae & Padina sp. & ++ \\
\hline & & & $\begin{array}{l}\text { Dictyota } \text { sp. } \\
\text { (Germling stage) }\end{array}$ & ++ \\
\hline & & Sargassaceae & $\begin{array}{l}\text { Sargassum ilicifolium } \\
\text { (Turner) C. Agardh }\end{array}$ & +++ \\
\hline \multirow{5}{*}{$\begin{array}{l}\text { Mayabandar, Middle } \\
\text { Andaman Island } \\
12^{\circ} 53^{\prime} \mathrm{N} \& 92^{\circ} 54^{\prime} \mathrm{E}\end{array}$} & $\begin{array}{l}\text { 30th January } 2005 \\
09: 00 \mathrm{~h}\end{array}$ & Sargassaceae & Sargassum sp.* & \\
\hline & & & $\begin{array}{l}\text { T. ornata } \\
\text { (Turner) J. Agardh* }\end{array}$ & +++ \\
\hline & & Gelidiaceae & $\begin{array}{l}\text { Gelidium pusillum } \\
\text { (Stackhouse) Le Jolis }\end{array}$ & ++ \\
\hline & & Ceramiaceae & Ceramium sp. & + \\
\hline & & Rhodomelaceae & $\begin{array}{l}\text { Bostrychia tenella } \\
\text { (Lamouroux) J. Agardh }\end{array}$ & +++ \\
\hline \multirow{2}{*}{$\begin{array}{l}\text { Rangat, Middle Andaman } \\
\text { Island } \\
12^{\circ} 32^{\prime} \mathrm{N} \& 92^{\circ} 58^{\prime} \mathrm{E}\end{array}$} & $\begin{array}{l}\text { 30th January } 2005 \\
09: 00 \mathrm{~h}\end{array}$ & Ulvaceae & Enteromorpha sp. & ++ \\
\hline & & Dictyotaceae & Dictyta sp. Lamouroux & + \\
\hline \multirow[t]{4}{*}{$\begin{array}{l}\text { Colachal, Tamil Nadu } \\
08^{\circ} 10^{\prime} \mathrm{N} \& 77^{\circ} 49^{\prime} \mathrm{E}\end{array}$} & $\begin{array}{l}\text { 8th January } 2005 \\
16: 00 \mathrm{~h}\end{array}$ & Ulvaceae & Ulva fasciata Delile & ++ \\
\hline & & Cladophoraceae & $\begin{array}{l}\text { Chaetomorpha } \\
\text { antennina (Bory de } \\
\text { Saint-Vincent) Kützing }\end{array}$ & +++ \\
\hline & & Sargassaceae & Sargassum sp. & +++ \\
\hline & & & Sargassum sp.* & + \\
\hline
\end{tabular}


Table 1. (Continued).

\begin{tabular}{|c|c|c|c|c|}
\hline \multirow[b]{2}{*}{ Locality } & \multirow{2}{*}{$\begin{array}{l}\text { Date and time } \\
\text { of sampling }\end{array}$} & \multicolumn{2}{|c|}{ Seaweed encountered } & \multirow{2}{*}{$\begin{array}{c}\text { Availability } \\
\text { status }\end{array}$} \\
\hline & & Family & Species & \\
\hline \multirow[t]{6}{*}{$\begin{array}{l}\text { Kanyakumari, Tamil Nadu } \\
08^{\circ} 40^{\prime} \mathrm{N} \& 77^{\circ} 36^{\prime} \mathrm{E}\end{array}$} & $\begin{array}{l}\text { 9th February } 2005 \\
6: 30 \mathrm{~h}\end{array}$ & Ulvaceae & $\begin{array}{l}\text { Enteromorpha } \\
\text { compressa (Linnaeus) } \\
\text { Nees }\end{array}$ & ++ \\
\hline & & & $\begin{array}{l}\text { Ulva fasciata Delile } \\
\text { Ulva fasciata Delile* }\end{array}$ & $\begin{array}{l}+++ \\
+\end{array}$ \\
\hline & & Cladophoraceae & $\begin{array}{l}\text { Chaetomorpha crassa } \\
\text { (C. Agardh) Kützing }\end{array}$ & + \\
\hline & & Caulerpaceae & $\begin{array}{l}\text { Caulerpa racemosa } \\
\text { (Forsskål) J. Agardh } \\
\text { Caulerpa racemosa } \\
\text { (Forsskål) J. Agardh* }\end{array}$ & $\begin{array}{l}++ \\
+\end{array}$ \\
\hline & & Dictyotaceae & $\begin{array}{l}\text { Dictyopteris delicatula } \\
\text { Lamouroux }\end{array}$ & +++ \\
\hline & & & $\begin{array}{l}\text { Lobophora variegata } \\
\text { (Lomouroux) } \\
\text { Womersley ex Oliveira } \\
\text { Lobophora variegata } \\
\text { (Lomouroux) } \\
\text { Womersley ex Oliveira* } \\
\text { Padina tetrastromatica } \\
\text { Hauck }\end{array}$ & $\begin{array}{l}+++ \\
++ \\
++\end{array}$ \\
\hline & & & $\begin{array}{l}\text { Padina tetrastromatica } \\
\text { Hauck* } \\
\text { Stoechospermum } \\
\text { marginatum } \\
\text { (C. Agardh) Kützing } \\
\text { Stoechospermum } \\
\text { marginatum } \\
\text { (C. Agardh) Kützing* }\end{array}$ & $\begin{array}{l}+ \\
+++ \\
++\end{array}$ \\
\hline & & Chnoosporaceae & $\begin{array}{l}\text { Chnoospora minima } \\
\text { (Hering) Papenfuss }\end{array}$ & +++ \\
\hline & & Sargassaceae & Sargassum sp. & +++ \\
\hline & & Gelidiaceae & Pterocladia sp. & + \\
\hline & & Gracilariaceae & $\begin{array}{l}\text { Gracilaria foliifera } \\
\text { (Forsskål) Børgesen } \\
\text { G. corticata (J. Agardh) } \\
\text { J. Agardh } \\
\text { G. debilis (Forsskål) } \\
\text { Børgesen } \\
\text { G. debilis (Forsskål) } \\
\text { Børgesen* }\end{array}$ & $\begin{array}{l}++ \\
+++ \\
+++ \\
+\end{array}$ \\
\hline & & Halymeniaceae & $\begin{array}{l}\text { Cryptonemia undulata } \\
\text { Sonder }\end{array}$ & +++ \\
\hline & & Rhizophyllidaceae & $\begin{array}{l}\text { Portieria hornemannii } \\
\text { (Lyngbye) P. Silva }\end{array}$ & ++ \\
\hline & & Hypneaceae & $\begin{array}{l}\text { Hypnea valentiae } \\
\text { (Turner) Montage } \\
\text { Hypnea valentiae } \\
\text { (Turner) Montage* }^{*}\end{array}$ & $\begin{array}{l}+++ \\
+++\end{array}$ \\
\hline & & Rhodymeniaceae & $\begin{array}{l}\text { Botryocladia leptopoda } \\
\text { (J. Agardh) Kylin }\end{array}$ & + \\
\hline & & Dasyaceae & $\begin{array}{l}\text { Dictyurus purpurascens } \\
\text { Bory de Saint-Vincent* }\end{array}$ & + \\
\hline
\end{tabular}


Table 1. (Continued).

\begin{tabular}{|c|c|c|c|c|}
\hline \multirow[b]{2}{*}{ Locality } & \multirow{2}{*}{$\begin{array}{l}\text { Date and time } \\
\text { of sampling }\end{array}$} & \multicolumn{2}{|c|}{ Seaweed encountered } & \multirow{2}{*}{$\begin{array}{l}\text { Availability } \\
\text { status }\end{array}$} \\
\hline & & Family & Species & \\
\hline \multirow{3}{*}{$\begin{array}{l}\text { Varakala, Kerala } \\
08^{\circ} 28^{\prime} \mathrm{N} \& 77^{\circ} 55^{\prime} \mathrm{E}\end{array}$} & 10th February 2005 & Ulvaceae & Enteromorpha sp. & \\
\hline & & & Ulva sp. & ++ \\
\hline & & Sargassaceae & $\begin{array}{l}\text { Sargassum_wightii } \\
\text { Greville* }\end{array}$ & + \\
\hline \multirow[t]{12}{*}{$\begin{array}{l}\text { Thirumullavarum, Kollam, } \\
\text { Kerala } \\
08^{\circ} 54^{\prime} \mathrm{N} \& 76^{\circ} 38^{\prime} \mathrm{E}\end{array}$} & $\begin{array}{l}\text { 11th February } 2005 \\
16: 30 \mathrm{~h}\end{array}$ & Ulvaceae & $\begin{array}{l}\text { Enteromorpha } \\
\text { thirumullavaramensis } \\
\text { Sulekha and Panikkar } \\
\text { Ulva lactuca Linneaus } \\
\text { U. fasciata Delile } \\
\text { U. fasciata Delile* } \\
\text { U. rigida } \text { C. Agardh }\end{array}$ & $\begin{array}{l}+++ \\
+ \\
++ \\
+ \\
+\end{array}$ \\
\hline & & Acrosiphoniaceae & $\begin{array}{l}\text { Acrosiphonia orientalis } \\
\text { (J. Agardh) P. Silva } \\
\text { Acrosiphonia orientalis } \\
\text { (J. Agardh) P. Silva* }\end{array}$ & $\begin{array}{l}+++ \\
+\end{array}$ \\
\hline & & Cladophoraceae & $\begin{array}{l}\text { Chaetomorpha } \\
\text { antennina (Bory de } \\
\text { Saint-Vincent) Kützing }\end{array}$ & ++ \\
\hline & & Valoniaceae & $\begin{array}{l}\text { Valoniopsis pachynema } \\
\text { (G. Martens) Børgesen }\end{array}$ & ++ \\
\hline & & Caulerpaceae & $\begin{array}{l}\text { Caulerpa peltata } \\
\text { Lamouroux } \\
\text { C. racemosa (Forsskål) } \\
\text { J. Agardh } \\
\text { C. sertularioides } \\
\text { (S. Gmelin) Howe } \\
\end{array}$ & $\begin{array}{l}+ \\
+++ \\
++\end{array}$ \\
\hline & & Ectocarpaceae & $\begin{array}{l}\text { Feldmannia indica } \\
\text { (Sonder) Womersley and } \\
\text { Bailey }\end{array}$ & + \\
\hline & & Dictyotaceae & $\begin{array}{l}\text { Lobophora variegata } \\
\text { (Lomouroux) } \\
\text { Womersley ex Oliveira } \\
\text { Padina gymnospora } \\
\text { (Kützing) Sonder } \\
\text { Stoechospermum } \\
\text { marginatum } \\
\text { (C. Agardh) Kützing } \\
\text { Stoechospermum } \\
\text { marginatum } \\
\text { (C. Agardh) Kützing* }\end{array}$ & $\begin{array}{l}++ \\
+++ \\
++ \\
+\end{array}$ \\
\hline & & Sargassaceae & $\begin{array}{l}\text { Sargassum tenerrimum } \\
\text { J. Agardh } \\
\text { Sargassum_sp. } \\
\text { Sargassum tenerrimum } \\
\text { J. Agardh* }\end{array}$ & $\begin{array}{l}+++ \\
++ \\
+\end{array}$ \\
\hline & & Gelidiellaceae & $\begin{array}{l}\text { Gelidiella acerosa } \\
\text { (Forsskål) J. Feldmann } \\
\text { and G. Hamel }\end{array}$ & + \\
\hline & & Gracilariaceae & $\begin{array}{l}\text { Gracilaria foliifera } \\
\text { (Forsskål) Børgesen }\end{array}$ & ++ \\
\hline & & & $\begin{array}{l}\text { Gracilaria foliifera } \\
\text { (Forsskål) Børgesen* }\end{array}$ & + \\
\hline & & Halymeniaceae & $\begin{array}{l}\text { Cryptonemia undulata } \\
\text { Sonder }\end{array}$ & +++ \\
\hline
\end{tabular}


Table 1. (Continued).

\begin{tabular}{|c|c|c|c|c|}
\hline \multirow[b]{2}{*}{ Locality } & \multirow{2}{*}{$\begin{array}{c}\text { Date and time } \\
\text { of sampling }\end{array}$} & \multicolumn{2}{|c|}{ Seaweed encountered } & \multirow{2}{*}{$\begin{array}{l}\text { Availability } \\
\text { status }\end{array}$} \\
\hline & & Family & Species & \\
\hline & & Rhizophyllidaceae & $\begin{array}{l}\text { Portieria hornemannii } \\
\text { (Lyngbye) P. Silva }\end{array}$ & +++ \\
\hline & & Corallinaceae & $\begin{array}{l}\text { Jania adhaerens } \\
\text { Lamouroux } \\
\text { Cheilosporum } \\
\text { spectabile Harvey ex } \\
\text { Grunow }\end{array}$ & $\begin{array}{l}++ \\
+\end{array}$ \\
\hline & & Hypneaceae & $\begin{array}{l}\text { Hypnea valentiae } \\
\text { (Turner) Montage } \\
\text { Hypnea valentiae } \\
\text { (Turner) Montage* }\end{array}$ & $\begin{array}{l}+++ \\
+\end{array}$ \\
\hline & & Rhodymeniaceae & $\begin{array}{l}\text { Gelidiopsis variabilis } \\
\text { (J. Agardh) Schmitz }\end{array}$ & ++ \\
\hline & & Ceramiaceae & $\begin{array}{l}\text { Ceramium cruciatum } \\
\text { Collins and Hervey }\end{array}$ & + \\
\hline & & Rhodomelaceae & Laurencia sp. & ++ \\
\hline \multirow{2}{*}{$\begin{array}{l}\text { Karaikal, Pondicherry } \\
10^{\circ} 55^{\prime} \mathrm{N} \& 79^{\circ} 52^{\prime} \mathrm{E}\end{array}$} & 15th February 2005 & Ulvaceae & Enteromorpha sp. & +++ \\
\hline & & Cladophoraceae & $\begin{array}{l}\text { Chaetomorpha aerea } \\
\text { (Dillwyn) Kützing }\end{array}$ & ++ \\
\hline \multirow{2}{*}{$\begin{array}{l}\text { Kodiakkarai (Point } \\
\text { Calimere) Tamil Nadu } \\
10^{\circ} 17^{\prime} \mathrm{N} \& 79^{\circ} 52^{\prime} \mathrm{E}\end{array}$} & 16th February 2005 & Ulvaceae & Enteromorpha sp. & + \\
\hline & & Cladophoraceae & Rhizoclonium sp. & + \\
\hline
\end{tabular}

Note: + meager, ++ average, +++ dominant; ${ }^{*}$ drift collection.

$\$$ Enteromorpha thirumullavaramensis species novo. Sulekha and Panikkar.

Source: Studies on the seasonal distribution and taxonomy of the marine green algae of Kerala, India, Ph.D. Thesis, University of Kerala, pp. 292.

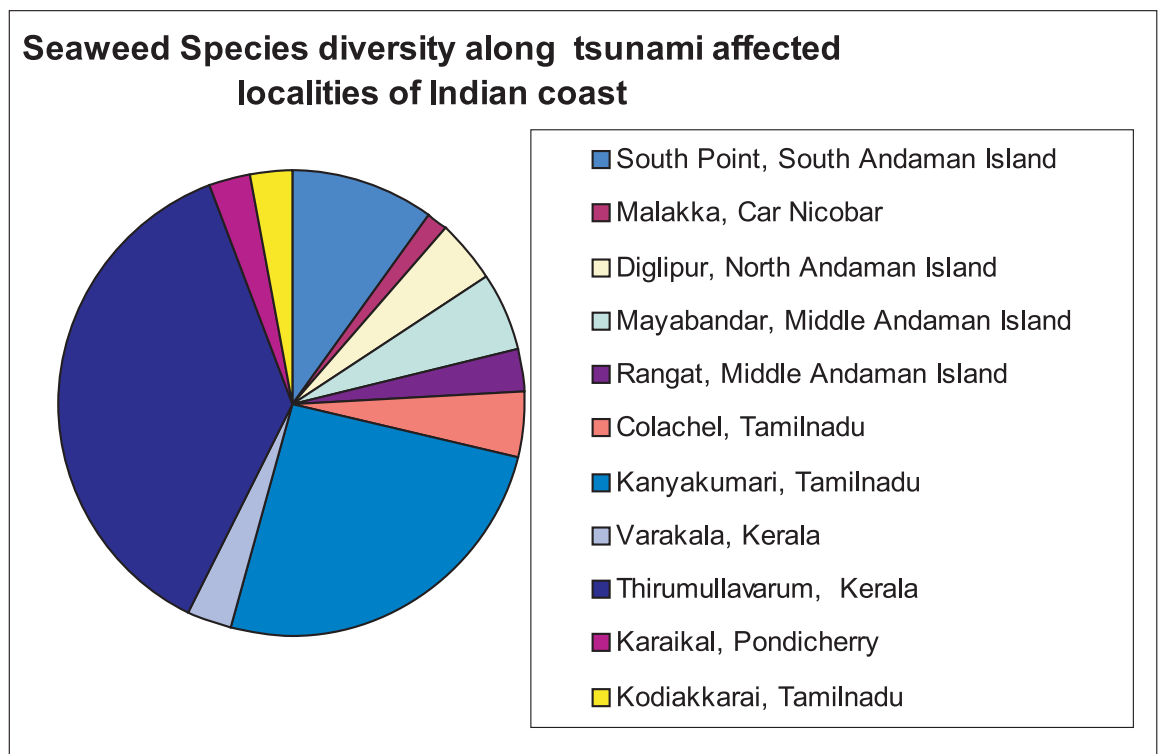

Figure 1. Seaweed diversity of affected places. 
Table 2. Biomass availability of seaweed species in some selected localities of tsunami affected regions of the Indian coast.

\begin{tabular}{|c|c|c|}
\hline Locality & Species & $\begin{array}{c}\text { Average biomass (SD) } \\
\text { g (dry) } \mathrm{m}^{-2}\end{array}$ \\
\hline \multirow{8}{*}{$\begin{array}{l}\text { South Point, Port Blair, } \\
\text { South Andaman Island }\end{array}$} & Ulva sp. & $0.97( \pm 1.40)$ \\
\hline & Enteromorpha flexuosa (Wulfen) & $2.88( \pm 2.22)$ \\
\hline & $\begin{array}{l}\text { J. Agardh } \\
\text { Chaetomorpha spiralis Okamura }\end{array}$ & $0.962( \pm 1.44)$ \\
\hline & Cladophora sp. & $2.22( \pm 1.68)$ \\
\hline & Lobophora variegata (Lomouroux) & $4.75( \pm 4.03)$ \\
\hline & Womersley ex Oliveira & \\
\hline & Padina tetrastromatica Hauck & $6.79( \pm 4.70)$ \\
\hline & Gracilaria canaliculata Sonder & $4.34( \pm 6.06)$ \\
\hline Malakka, Car Nicobar & Enteromorpha compressa (Linnaeus) Nees & $6.98( \pm 4.76)$ \\
\hline \multirow{3}{*}{$\begin{array}{l}\text { Diglipur, North Andaman } \\
\text { Island }\end{array}$} & Padina sp. & $13.44( \pm 8.98)$ \\
\hline & Dictyota sp. & $15.15( \pm 10.73)$ \\
\hline & Sargassum ilicifolium (Turner) C. Agardh & $27.92( \pm 13.14)$ \\
\hline \multirow[t]{3}{*}{ Colachal, Tamil Nadu } & Ulva fasciata Delile & $14.24( \pm 6.27)$ \\
\hline & $\begin{array}{l}\text { Chaetomorpha antennina (Bory de } \\
\text { Saint-Vincent) Kützing }\end{array}$ & $6.46( \pm 1.97)$ \\
\hline & Sargassum sp. & $10.67( \pm 10.63)$ \\
\hline \multirow[t]{18}{*}{ Kanyakumari, Tamil Nadu } & Enteromorpha compressa (Linnaeus) Nees & $22.05( \pm 7.98)$ \\
\hline & Ulva fasciata Delile & $33.33( \pm 16.31)$ \\
\hline & Chaetomorpha crassa (C. Agardh) Kützing & $15.23( \pm 9.82)$ \\
\hline & Caulerpa racemosa (Forsskål) J. Agardh & $147.65( \pm 69.26)$ \\
\hline & Dictyopteris delicatula Lamouroux & $9.63( \pm 9.44)$ \\
\hline & $\begin{array}{l}\text { Lobophora variegata (Lomouroux) } \\
\text { Womersley ex Oliveira }\end{array}$ & $41.01( \pm 9.89)$ \\
\hline & Padina tetrastromatica Hauck & $107.54( \pm 95.03)$ \\
\hline & $\begin{array}{l}\text { Stoechospermum marginatum (C. Agardh) } \\
\text { Kützing }\end{array}$ & $10.08( \pm 6.35)$ \\
\hline & Chnoospora minima (Hering) Papenfuss & $31.69( \pm 44.87)$ \\
\hline & Sargassum sp. & $316.88( \pm 88.75)$ \\
\hline & Pterocladia sp. & $12.39( \pm 12.43)$ \\
\hline & Gracilaria foliifera (Forsskål) Børgesen & $129( \pm 132.77)$ \\
\hline & G. corticata (J. Agardh) J. Agardh & $88.67( \pm 108.30)$ \\
\hline & G. debilis (Forsskål) Børgesen & $3.16( \pm 6.82)$ \\
\hline & Cryptonemia undulata Sonder & $17.8( \pm 39.80)$ \\
\hline & Portieria hornemannii (Lyngbye) P. Silva & $3.02( \pm 2.81)$ \\
\hline & Hypnea valentiae (Turner) Montage & $149.254( \pm 37.37)$ \\
\hline & Botryocladia leptopoda (J. Agardh) Kylin & $9.74( \pm 7.87)$ \\
\hline \multirow[t]{2}{*}{ Varakala, Kerala } & Enteromorpha sp. & $6.61( \pm 6.13)$ \\
\hline & Ulva sp. & $12.88( \pm 7.40)$ \\
\hline \multirow[t]{11}{*}{$\begin{array}{l}\text { Thirumullavarum, Kollam, } \\
\text { Kerala }\end{array}$} & $\begin{array}{l}\text { Enteromorpha thirumullavaramensis } \\
\text { Sulekha and Panikkar }\end{array}$ & $6.88( \pm 4.09)$ \\
\hline & Ulva lactuca Linneaus & $14.56( \pm 8.39)$ \\
\hline & U. fasciata Delile & $39.36( \pm 27.46)$ \\
\hline & U. rigida C. Agardh & $14.14( \pm 2.68)$ \\
\hline & $\begin{array}{l}\text { Acrosiphonia orientalis (J. Agardh) } \\
\text { P. Silva }\end{array}$ & $17.12( \pm 9.90)$ \\
\hline & $\begin{array}{l}\text { Chaetomorpha antennina (Bory de Saint- } \\
\text { Vincent) Kützing }\end{array}$ & $13.08( \pm 9.21)$ \\
\hline & Valoniopsis pachynema (G. Martens) & $24.11( \pm 23.60)$ \\
\hline & Børgesen & \\
\hline & Caulerpa peltata Lamouroux & $41.42( \pm 29.35)$ \\
\hline & C. racemosa (Forsskål) J. Agardh & $7.18( \pm 5.52)$ \\
\hline & C. sertularioides (S. Gmelin) Howe & $15.64( \pm 24.03)$ \\
\hline
\end{tabular}


Table 2. (Continued).

\begin{tabular}{|c|c|c|}
\hline Locality & Species & $\begin{array}{c}\text { Average biomass (SD) } \\
\mathrm{g}(\text { dry }) \mathrm{m}^{-2}\end{array}$ \\
\hline & $\begin{array}{l}\text { Feldmannia indica (Sonder) Womersley } \\
\text { and Bailey }\end{array}$ & $1.01( \pm 1.14)$ \\
\hline & Lobophora variegata (Lomouroux) & $7.36( \pm 7.04)$ \\
\hline & Womersley ex Oliveira & \\
\hline & Padina gymnospora (Kützing) Sonder & $22.47( \pm 19.66)$ \\
\hline & $\begin{array}{l}\text { Stoechospermum marginatum (C. Agardh) } \\
\text { Kützing }\end{array}$ & $10.69( \pm 3.96)$ \\
\hline & Sargassum tenerrimum J. Agardh & $27.20( \pm 35.01)$ \\
\hline & Sargassum sp. & $130.54( \pm 42.94)$ \\
\hline & $\begin{array}{l}\text { Gelidiella acerosa (Forsskål) J. Feldmann } \\
\text { and G. Hamel }\end{array}$ & $13.69( \pm 13.13)$ \\
\hline & Gracilaria foliifera (Forsskål) Børgesen & $18.16( \pm 4.32)$ \\
\hline & Cryptonemia undulata Sonder & $1.24( \pm 1.89)$ \\
\hline & Portieria hornemannii (Lyngbye) P. Silva & $0.57( \pm 0.80)$ \\
\hline & Jania adhaerens Lamouroux & $3.83( \pm 3.84)$ \\
\hline & $\begin{array}{l}\text { Cheilosporum spectabile Harvey ex } \\
\text { Grunow }\end{array}$ & $0.916( \pm 2.04)$ \\
\hline & Hypnea valentiae (Turner) Montage & $6.87( \pm 2.87)$ \\
\hline & Gelidiopsis variabilis (J. Agardh) Schmitz & $0.89( \pm 1.24)$ \\
\hline & Ceramium cruciatum Collins and Hervey & $0.26( \pm 0.39)$ \\
\hline & Laurencia sp. & $0.25( \pm 0.55)$ \\
\hline \multirow{2}{*}{$\begin{array}{l}\text { Kodiakkarai (Point } \\
\text { Calimere) Tamil Nadu }\end{array}$} & Enteromorpha sp. & $0.312( \pm 0.69)$ \\
\hline & Rhizoclonium sp. & $0.09( \pm 0.20)$ \\
\hline
\end{tabular}

Table 3. Species similarity index for selected tsunami affected location of the Indian coast.

\begin{tabular}{lc}
\hline Locations & Similarity index \\
\hline South Point, Port Blair vs. Kanyakumari & 0.077 \\
South Point, Port Blair vs. Thirumullavarum & 0.030 \\
Kanyakumari vs. Thirumullavarum & 0.157 \\
\hline
\end{tabular}

study were identified, recorded and classified as meager $(+)$, average $(++)$ and dominant $(+++)$ based on their occurrence in the natural habitat. Few of the sample materials were preserved in $4 \%$ formaldehyde in seawater for further critical observation. The biomass availability was recorded for eight coastal locations viz., South Point $\left(11^{\circ} 39^{\prime} \mathrm{N} \& 92^{\circ} 45^{\prime} \mathrm{E}\right)$, Port Blair, south Andaman Island; Malakka (09 $\left.10^{\prime} \mathrm{N} \& 92^{\circ} 49^{\prime} \mathrm{E}\right)$, Car Nicobar; Diglipur $\left(11^{\circ} 39^{\prime} \mathrm{N} \& 92^{\circ} 45^{\prime} \mathrm{E}\right)$, north Andaman Island; Colachal $\left(08^{\circ} 10^{\prime} \mathrm{N}\right.$ \& $\left.77^{\circ} 49^{\prime} \mathrm{E}\right)$, Tamil Nadu; Kanyakumari $\left(08^{\circ} 40^{\prime} \mathrm{N}\right.$ \& $\left.77^{\circ} 36^{\prime} \mathrm{E}\right)$, Tamil Nadu; Varakala $\left(08^{\circ} 28^{\prime} \mathrm{N} \&\right.$ $\left.76^{\circ} 55^{\prime} \mathrm{E}\right)$, Kerala; Thirumullavarum (08 $54^{\prime} \mathrm{N}$ \& $76^{\circ} 38^{\prime}$ E) Kollam, Kerala; Kodiakkarai $\left(10^{\circ} 17^{\prime} \mathrm{N}\right.$ \& $79^{\circ} 52^{\prime}$ E) Tamil Nadu by placing 5 random quadrates of $0.5 \mathrm{~m}^{2}$ size. Seaweeds collected from these quadrates were brought to a laboratory washed thoroughly to make them free from sediments and epiphytic animals, air dried followed by hot oven drying at $60^{\circ} \mathrm{C}$ for $48 \mathrm{~h}$ and the biomass was then estimated as ' $\mathrm{g}$ dry weight $\mathrm{m}^{2}$ '. The voucher specimens were given accession numbers and deposited at 'Marine Algae Herbarium', Central Salt and Marine Chemicals Research Institute, Bhavnagar, India as reference material. The species similarity index (Margalef 1968) for selected localities was calculated for comparison of seaweed diversity.

\section{Observations and discussion}

Though many places along eastern coastal India have been affected during the recent tsunami, Andaman and Nicobar and coastal Tamil Nadu and Kerala, are of immediate concern since they are rich in seaweed wealth (Krishnamurthy et al 1980; Krishnamurthy 1991; Nair et al 1986; Rao 2000). On-the-spot verification at all the 11 localities revealed the presence of 15 species of seaweeds belonging to 14 genera of Rhodophyta, 
15 species to 9 genera of Phaeophyta and 15 species to 8 genera of Chlorophyta (table 1). Maximum species diversity was recorded at Thirumullavarum coast, Kerala with the least from Car Nicobar, Andaman and Nicobar Islands (figure 1). The high seaweed floristic diversity (24 seaweed species, $32.43 \%$ ) found at Thirumullavarum coast could be ascribed to the least tsunami damage in this area and less diverse flora (only 1 seaweed species, $1.35 \%$ ) observed at Car Nicobar, could be the result of supercilious damage here. However, the influence of habitat suitability over tsunami damage was more pronounced at some localities, which was reflected in biomass availability viz., rich seaweed growth at Kanyakumari [Sargassum sp. recorded $316.88 \pm 88.75 \mathrm{~g}$ (dry) $\mathrm{m}^{-2}$ ] and poor seaweed growth at Kodiakkarai (Point Calimere) $\left[0.09 \pm 0.20 \mathrm{~g}(\mathrm{dry}) \mathrm{m}^{-2}\right]$ (table 2). The comparison of seaweed flora at South Point, Port Blair, south Andaman Island, Kanyakumari and Thirumullavarum showed comparatively higher value for Kanyakumari vs. Thirumullavarum (table 3 ). The dissimilarity of this biological variable is obvious and could be related to the special variation and environmental, physico-chemical and geographical features at these locations (Anonymous 1978).

In conclusion, to understand the long-term ecological consequences due to the recent Asian tsunami, we need to have more detailed studies. Immediate assessment of the loss of seaweed biodiversity at the localities under study was not possible since the corresponding data prior to tsunami for these localities were not available. Though some information for a few affected places is available, (Srinivasan 1969, 1973; Krishnamurthy and Joshi 1970; Nair et al 1982; Chennubhotla et al 1991; Jagtap 1992; Chennubhotla 1992; Kaliaperumal and Kalimuthu 1993) it is too old and fragmentary for any genuine comparison. The present study is in no way extensive and complete; but still it will be useful and could be taken as new baseline record for future biomonitoring studies.

\section{Acknowledgements}

Thanks are due to Dr. P K Ghosh, Director, for his constant encouragement during the entire course of this study. Thanks are also due to Prof. B Jha, for extending necessary facilities. The author is indebted to Dr. P G Diwakar, Dr. M V N Panikkar, and Dr. K Eswaran, for their support during field studies. Special thanks are due to Prof. V Krishnamurthy, Founder Director, KIA for his help in the identification of some seaweeds and to Dr. P V Subba Rao, for his constructive suggestions. The author would also like to acknowledge Prof. L Kannan, Director - Research, Center of Advanced Studies in Marine Biology, Annamalai University, Parangipettai and an anonymous referee for their comments on improving the manuscript.

\section{References}

Anonymous 1978 A report on the survey of marine algal resources of Tamil Nadu, 1971-1976; Central Salt and Marine Chemicals Research Institute, Bhavnagar, pp. 137.

Chennubhotla V S K 1992 Survey of seaweed resources of Andaman Nicobar Islands, Visakhapatanam - Chilka lake region and Lakshadweep group of Islands, Final Technical report ICAR - Ad-hoc Scheme.

Chennubhotla V S K, Kaliaperumal N, Kalimuthu S, Ramalingam J, Subhramaih K, Rama Rao K and Subba Rao P V 1991 Seaweed resources of the Tuticorin Tiruchendur coast, Tamil Nadu; J. Mar. Bio. Assoc. India 32 146-149.

Jagtap T G 1992 Marine flora of Nicobar group of Islands in Andaman Sea; Indian J. Mar. Sci. 21 56-58.

Jain S K, Murty C V R, Rai D C, Malik J N, Sheth A and Jaiswal A 2005 Effects of M 9 Sumatra earthquake and tsunami of 26 December 2004; Curr. Sci. 88 357-356.

Kaliaperumal N and Kalimuthu S 1993 Need for conservation of economically important seaweeds of Tamil Nadu coast and time table for commercial exploitation, Mar. Fish. Info. Service, Technical Series 119 5-12.

Krishnamurthy V 1991 Gracilaria resources of India with particular reference to Tamil Nadu coast; Seaweed Res. Utiln. 14 1-7.

Krishnamurthy V and Joshi H V 1970 A checklist of Indian marine algae, Central Salt and Marine Chemicals Research Institute, Bhavnagar, pp. 137.

Krishnamurthy V, Raju P V and Thomas P C 1980 On augmenting seaweed resources of India; J. Mar. Biol. Assoc. India 17 181-185.

Mantri V A 2005 Changes in local intertidal seaweed habitats in the Andaman \& Nicobar Islands after 26 December 2004 tsunami; Curr. Sci. 89 1071-1072.

Margalef R 1968 Perspectives in Ecological Theory; (Chicago: University of Chicago Press) pp. 112.

Nair N B, Sobha V and Arunachalam M 1982 Algae from southern Kerala coast; Indian J. Mar. Sci. 11 266-269.

Nair N B, Sobha V, Chandran R, Rathiamma M, Maya S and Suryanarayanan H 1986 Nature and distribution of the littoral algae and sea grasses of the southwest coast of India; Proc. Indian Nat. Sci. Acad. Part B: Bio. Sci. $52733-744$.

Rao U M 2000 Some Marine Algae from Andaman and Nicobar Island; Phycos 39 85-99.

Sahoo D, Sahu N and Sahoo D 2001 Seaweeds of Indian Coast; (New Delhi: A. P. H. Publishing Corporation) pp. 283.

Srinivasan K S 1969 Phycologia Indica, Vol. I, Botanical Survey of India, Culcutta, pp. 52.

Srinivasan K S 1973 Phycologia Indica, Vol. II, Botanical Survey of India, Culcutta, pp. 60. 